\title{
Demostración de afecto materno y paternos la experiencia de adolescentes
}

Demonstration of paternal and maternal affection: Adolescent experience

\author{
Georgina Lozano Razo \\ Javier Zavala Rayas \\ Silvia del Carmen Miramontes Zapata \\ Juan Martín Sánchez Bautista \\ María Dolores Jiménez López \\ Francisco Pacheco Medina \\ María Dolores García Sánchez \\ Oliva Erendira Luis Delgado
}

\section{Resumen}

El objetivo de la presente investigación fue describir la experiencia de hombres y mujeres adolescentes de 15 a 18 años, que habitan en el estado de Zacatecas, respecto a la demostración de afecto paterno y materno. Para ello se realizó un muestreo por cuotas, por nivel escolar de secundaria y bachillerato y género; se obtuvo una muestra total de 80 adolescentes, a los cuales se les pidió que contestaran dos preguntas: ¿Qué hace tu mamá para demostrarte afecto? y ¿qué hace tu papá para demostrarte afecto? Las respuestas fueron sometidas a un análisis de contenido. Se encontró que para los varones, las categorías más comunes de afecto materno, fueron: expresión física de afecto, expresión verbal de afecto, cuidados y protección y expresión material de afecto. Mientras que para el afecto paterno fueron: expresión material de afecto, pasar tiempo con ellos y expresión física de afecto. Por su parte, para las adolescentes, las categorías más comunes de afecto materno, fueron: expresión física de afecto, expresión verbal de afecto y cuidados y protección. Mientras que las de afecto paterno, fueron: expresión física de afecto, expresión verbal de afecto y no demuestra su afecto.

\section{Abstract}

The aim of this study was to describe the experience of male and female adolescents from 15 to 18 years old, who live in the state of Zacatecas, regarding the demonstration of paternal and maternal affection. 80 adolescents who were chosen by a quota sampling, school years (middle and high school), and gender were asked two questions. How does your mom show you affection? and how does your dad show you affection? The answers were subjected to content analysis. The findings for males showed that the most common categories of affection from their mother were: physical expression of affection, verbal affection, care and protection and material expression of affection. On the other hand, affection categories from their fathers were material expression of affection, spending time with them and physical expression of affection. For teenage girls, the most common categories from their mother's affection were: physical expression of affection, verbal expression of affection and care and protection. And from their fathers were: physical expression of affection, verbal expression of affection, and no signs of affection.

Palabras Clave: Crianza, padres, afecto, adolescentes

Keywords: Parenting, parents, affection, adolescence 


\section{Introducción}

Cuando los padres se relacionan con los hijos y realizan sus funciones, ponen en práctica una serie de tácticas que tiene la finalidad de influir, educar y orientar a los hijos para su integración social, es por ello que se relacionan con dimensiones como el tipo de disciplina, el tono de la relación, el mayor o menor nivel de comunicación y las formas que adopta la expresión del afecto (Ramírez, 2005). Por su parte, Oliva, Parra y Arranz (2008) mencionan que la característica distintiva de dichas tácticas es alentar o desalentar comportamientos específicos enmarcados en las relaciones familiares, en las cuales es importante el papel que juegan los padres en la formación de sus hijos. Por otro lado, se evidencia que los padres tienen una noción espontánea, no muy elaborada, de la manera cómo se debe criar a los hijos y son capaces de desarrollar teorías sobre la mejor forma de realizar esta tarea.

En este sentido, en 1968, Baumrind acuñó el concepto de estilo parental y propuso una tipología de tres estilos parentales (democrático, autoritario y permisivo). El concepto de estilo parental ha sido ampliamente utilizado en la investigación acerca de los efectos de la socialización familiar sobre la competencia de niños y adolescentes. Posteriormente, en 1983, Maccoby y Martin enriquecieron la propuesta de Baumrind, proponiendo un cuarto estilo, el indiferente. Esta tipología se basa en el cruce de dos dimensiones fundamentales: afecto y control. Sin embargo, la evidencia empírica acumulada por años de investigación, demuestra que el estilo parental es un concepto multidimensional y que se deben tomar en consideración otras variables, además del afecto y el control (Oliva et al., 2008).

Musitu, Román y Gracia (1988) al considerar los factores que determinan dicho estilo, señalan los que contribuyen a una mejor práctica educativa como son la estructura, afecto, control conductual, comunicación, transmisión de valores y sistemas externos. Los cuatro primeros hacen referencia a las relaciones intrafami- liares o microsistémicas; los últimos se refieren a la dimensión social o ecológica. La posición dentro de un sistema más amplio explicará en gran medida la toma de postura y modos de actuación del grupo social que es la familia.

De acuerdo con Collins y Laursen (2004) las conductas parentales influyen sobre el desarrollo del adolescente, pero también es cierto que los comportamientos y actitudes del adolescente tienen impacto en el estilo parental. La literatura sobre los efectos de los diferentes estilos parentales en el desarrollo de niños, niñas y adolescentes es abundante; por ejemplo, se ha demostrado la importancia que tiene el afecto y la comunicación sobre el bienestar y el ajuste de los individuos. Los trabajos que abordan el estudio de la influencia del afecto, concluyen que chicos y chicas que afirman tener relaciones más cercanas con sus padres y madres manifiestan un mejor desarrollo psicosocial, un mayor bienestar emocional y un ajuste más positivo a nivel interno y externo (Gray \& Steinberg, 1999; Oliva, Parra, \& Sánchez-Queija, 2002; Dusek \& McIntyre, 2003; Parra, Oliva, \& Sánchez-Queija, 2004).

El afecto ha sido una de las variables del contexto familiar más analizadas cuando se intenta entender el bienestar de los hijos. Parra y Oliva (2006) realizaron un análisis longitudinal para describir la evolución que siguen a lo largo de la adolescencia cuatro dimensiones de los estilos parentales, entre ellas el afecto. Los resultados indican que en general existen pocos cambios a lo largo de los años; aunque algunos jóvenes señalan cierta disminución entre la adolescencia media y tardía, la mayoría describe unos niveles semejantes en los diferentes momentos.

Recordemos que la adolescencia se caracteriza por cambios drásticos y rápidos en el desarrollo físico, cognitivo y emocional, que afectan las relaciones de los adolescentes con sus padres y con los iguales (Rodrigo et al., 2004). De acuerdo con Motrico, Fuentes y Bersabé (2001) estos cambios no se originan de forma brus- 
ca y rápida, sino de manera progresiva y continuada, lo cual permite que tanto padres como adolescentes se vayan adaptando a la nueva situación. Para estos autores es más adecuado hablar de transformaciones en las relaciones familiares durante la adolescencia. Aunque se ha encontrado que estas transformaciones pueden dar lugar a conflictos en las relaciones familiares, los estudios actuales revelan que la adolescencia no es una etapa tan turbulenta como se pensaba en épocas anteriores (Oliva, 2006).

De acuerdo con Oliva (2006), en la adolescencia la interacción entre padres e hijos debe acomodarse a las importantes transformaciones que experimentan los hijos, y pasará de la marcada jerarquización propia de la niñez, a la mayor igualdad y equilibrio de poder que caracterizan las relaciones parentofiliales durante la adolescencia tardía y la adultez emergente. La escasa presencia física o accesibilidad de los padres y la falta de supervisión de éstos, acompañada por una ausencia de comunicación con los hijos en relación con las actividades de la vida diaria, se asocian a una mayor tendencia de éstos a relacionarse con iguales conflictivos y a realizar conductas de riesgo o de carácter antisocial (Dishion, Patterson, Stoolmiller \& Skinner, 1991; Serrano, Godás, Rodríguez \& Mirón, 1996). Por el contrario, un mayor apoyo percibido de la familia se asocia a un menor consumo de tabaco, alcohol (Martínez \& Robles, 2001) y drogas en el adolescente, incluso cuando su grupo de iguales presenta un consumo extremo (Frauenglass, Routh, Pantin \& Mason, 1997).

Por su parte, Oliva (2006) menciona que el afecto es la dimensión más relevante a la hora de definir las relaciones entre padres y adolescentes. Al utilizar el término para hacer referencia a aspectos como la cercanía emocional, el apoyo, la armonía o la cohesión, esta dimensión aparece asociada al control o monitorización en la definición que Baumrind (1968) realizó del estilo parental democrático. Aunque puede considerarse una dimensión diferente, la comunicación muestra una fuerte asociación con el afecto. Cabe la pena destacar que el afecto y la comunicación muestran una continuidad presencial en las relaciones parentofiliales durante la infancia y la adolescencia, ya que aquellos niños y niñas que sostienen intercambios cálidos y afectuosos con sus padres son quienes mantienen una relación más estrecha con ellas cuando llega la adolescencia. Sin embargo, esa continuidad coexiste con cambios significativos en las interacciones, tanto en las expresiones positivas y negativas de afecto como en la percepción que unos y otros tienen de su relación (Flouri \& Buchanan, 2002).

Existen datos según los cuales durante la adolescencia se presenta una disminución de la cercanía emocional, de las expresiones de afecto y de la cantidad de tiempo que padres e hijos pasan juntos. La comunicación también suele experimentar un ligero deterioro en torno a la pubertad, ya que en esta etapa los hijos hablan menos espontáneamente de sus asuntos, las interrupciones son más frecuentes y la comunicación se hace más difícil. No obstante, tal deterioro suele ser pasajero, y en la mayoría de familias la comunicación, al igual que el afecto positivo, suele recuperarse a lo largo de la adolescencia. Se han observado ligeras diferencias en cuanto al género en los niveles globales de afecto y comunicación: las chicas se sitúan por encima de los chicos en todas las edades, la disminución de afecto y comunicación seguida de la posterior recuperación suele darse de forma similar en ambos sexos (Parra \& Oliva, 2002). Estas diferencias coinciden con las de otros trabajos (von der Lippe, 1998), lo que puede estar reflejando prácticas de socialización distintas hacia chicos y chicas.

De acuerdo con Oliva (2006) el afecto se puede considerar como la dimensión clave del estilo democrático durante la adolescencia, ya que muestra una asociación muy significativa y poco controvertida con el desarrollo y ajuste en esta etapa. Dicha estrecha relación no se 
ve afectada por el contexto cultural, como puso de manifiesto el meta-análisis de Khaleque y Rohner (2002) sobre muestras de 43 estudios realizados en los cinco continentes, donde se encontró que el afecto explicaba $26 \%$ de la varianza en el ajuste de niños y adolescentes.

A pesar del relativo distanciamiento afectivo y comunicativo que se producirá en muchas díadas con la llegada de la adolescencia, los hijos van a seguir beneficiándose de unos padres comunicativos, cercanos y afectuosos, que les apoyen en los momentos difíciles que atravesarán a lo largo de estos años. Cuando el afecto, el apoyo y la comunicación positiva caracterizan las relaciones entre padres y adolescentes, estos últimos muestran un mejor ajuste psicosocial, incluyendo confianza en sí mismos, competencia conductual y académica, autoestima y bienestar psicológico, menos síntomas depresivos y menos problemas comportamentales. Además, es más probable que los hijos se muestren receptivos a los intentos socializadores por parte de sus padres y no se rebelen ante sus estrategias de control cuando existe un clima emocional favorable (Oliva et al., 2002).

De acuerdo con los resultados encontrados por Martínez, Fuertes, Ramos y Hernández (2003) en un estudio realizado para conocer la importancia del afecto y supervisión parental sobre el consumo de drogas, los adolescentes que perciben mayor afecto/apoyo y supervisión/ control por parte de sus padres, se involucran en menos conductas de riesgo asociadas al consumo de drogas. Por otra parte, las relaciones tanto con la madre como con el padre, así como la implicación de ambos padres en la crianza, la disponibilidad y el grado de apoyo que recibe el adolescente, y en general, el predominio de una buena comunicación, proporcionan un buen apoyo instrumental y emocional para los hijos. Además, los padres que transmiten apoyo y afecto a sus hijos, desarrollan la comunicación en el ámbito familiar, establecen normas familiares y el cumplimiento de las mismas utilizando el razonamiento inductivo como técnica de disciplina, educan con mayor probabilidad hijos sociables, cooperativos y autónomos (Mestre, Tur, Samper, Nácher \& Cortés, 2007).

Las dimensiones de control, afecto y grado de implicación en la crianza por parte de los padres constituyen pilares fundamentales en la educación de los hijos y son las variables que mejor predicen un buen estilo educativo y calidad de las relaciones paterno-filiales. Estos factores inciden sobre la cohesión familiar, el grado de adaptabilidad y la calidad de la comunicación entre los miembros de la misma familia, elementos que pueden favorecer o entorpecer tanto la cohesión como la adaptabilidad (Mestre, Frías, Samper \& Nácher, 2003). Es por ello que en la presente investigación el objetivo fue describir la experiencia que viven hombres y mujeres adolescentes de 15 a 18 años, que habitan en el estado de Zacatecas, respecto a la demostración de afecto paterno y materno.

\section{Método}

Se realizó un estudio descriptivo, cuyo propósito fue seleccionar una serie de cuestiones y medir cada una de ellas independientemente, para de esta forma describir lo que se investiga (Hernández, Fernández \& Baptista, 2008).

\section{Participantes}

Los participantes fueron 80 adolescentes de ambos sexos ( 40 mujeres y 40 hombres) de las ciudades de Zacatecas y Fresnillo, Zacatecas, con edades entre los 15 y 18 años. Todos los participantes cursaban el bachillerato en el momento de la aplicación. La muestra fue de tipo no probabilístico intencional. 


\section{Instrumento}

Se aplicó un cuestionario de preguntas abiertas que explora las prácticas de crianza a través de las acciones que los padres realizan - llevan a cabo en la educación de sus hijos. El cuestionario fue elaborado por un grupo de investigadores que lleva a cabo el proyecto de investigación "Crianza, personalidad y adolescencia". El cuestionario consta de una sección de datos generales y 2 preguntas relacionadas con la demostración de afecto por parte de papá y mamá hacia el hijo/a adolescente (¿qué hace tu mamá para demostrarte afecto? y ¿qué hace tu papá para demostrarte afecto?).

\section{Procedimiento}

Las aplicaciones se llevaron a cabo en escuelas de nivel bachillerato; una vez que se obtuvo el permiso de las autoridades correspondientes, se procedió a realizar una aplicación colectiva de los cuestionarios en los salones de clase. Se les explicó a los estudiantes que se solicitaba su colaboración para contestar un cuestionario acerca de cómo era la relación con sus padres, por lo que aquellos que accedieran a colaborar podían levantar la mano para proporcionarles el cuestionario. Posteriormente, se procedió a dar las instrucciones para que pudieran contestar.

\section{Análisis de datos}

Se aplicó estadística descriptiva, frecuencias y porcentajes para los datos generales (edad, número de hermanos y con quién vive). Las preguntas que se refieren a la demostración de afecto por parte de los padres ¿qué hace tu mamá para demostrarte afecto? y ¿qué hace tu papá para demostrarte afecto? fueron sometidas a un análisis de contenido temático.

\section{Resultados}

Respecto a los datos generales de los participantes, la edad promedio fue de 16 años, tanto para mujeres como para hombres; asimismo, para ambos el promedio de hermanos fue de dos. El $73.2 \%$ de las mujeres vive con ambos padres y en el caso de los hombres el $84.8 \%$ (tabla 1).

En la tabla 2 se observan las categorías obtenidas en hombres y mujeres, así como ejemplos de respuestas y porcentaje para cada categoría para la pregunta ¿qué hace tu mamá para demostrarte afecto? Como puede observarse, en el caso de los adolescentes, las categorías con porcentajes más altos son para la expresión física y verbal de afecto y para la categoría de comunicación/tiempo, mientras que las categorías con porcentajes más bajos son el respeto, consejos y juego.

En el caso de las adolescentes la categoría de expresión física de afecto presenta el porcentaje más alto, seguida de la expresión verbal de afecto y después la de cuidados y protección. Las categorías con porcentajes más bajos son respeto y juego. Cabe señalar que a pesar de que las categorías expresión física de afecto y expresión verbal de afecto obtuvieron los porcentajes más altos tanto en hombres como en mujeres, en el caso de estas últimas, los porcentajes son mayores en ambas. Por su parte los adolescentes no reportaron respuesta alguna para la enseñanza de valores a diferencia de las chicas que sí lo hicieron.

Finalmente, en la tabla 3 se observan las categorías obtenidas en hombres y mujeres, así como ejemplos de respuestas y porcentaje para cada categoría para la pregunta ¿qué hace tu papá para demostrarte afecto? En el caso de los varones, las dos categorías con 
Tabla 1.

Datos generales de hombres y mujer

\begin{tabular}{|c|c|c|c|c|c|}
\hline \multicolumn{2}{|c|}{ Hombres } & \multicolumn{3}{|c|}{ Mujeres } & \multirow[b]{2}{*}{ Porcentaje } \\
\hline Edad & Frecuencia & Porcentaje & Edad & Frecuencia & \\
\hline 15 & 15 & $37.5 \%$ & 15 & 9 & $22.5 \%$ \\
\hline 16 & 14 & $35.0 \%$ & 16 & 20 & $50.0 \%$ \\
\hline 17 & 5 & $12.5 \%$ & 17 & 10 & $25.0 \%$ \\
\hline 18 & 6 & $15.0 \%$ & 18 & 1 & $2.5 \%$ \\
\hline Hermanos menores & Frecuencia & Porcentaje & Hermanos menores & Frecuencia & Porcentaje \\
\hline 0 & 10 & $25.0 \%$ & & & \\
\hline 1 & 14 & $35.0 \%$ & & & \\
\hline 2 & 10 & $25.0 \%$ & & & \\
\hline 3 & 3 & $7.5 \%$ & & & \\
\hline 4 & 3 & $7.5 \%$ & & & \\
\hline Hermanos mayores & Frecuencia & Porcentaje & Hermanos mayores & Frecuencia & Porcentaje \\
\hline 0 & 13 & $32.5 \%$ & 0 & 15 & $37.5 \%$ \\
\hline 1 & 12 & $30.0 \%$ & 1 & 12 & $30.0 \%$ \\
\hline 2 & 6 & $15.0 \%$ & 2 & 8 & $20.0 \%$ \\
\hline 3 & 3 & $7.5 \%$ & 3 & 4 & $10.0 \%$ \\
\hline 4 & 2 & $5.0 \%$ & 5 & 1 & $2.5 \%$ \\
\hline 5 & 2 & $5.0 \%$ & & & \\
\hline 6 & 2 & $5.0 \%$ & & & \\
\hline Vive con: & Frecuencia & Porcentaje & Vive con: & Frecuencia & Porcentaje \\
\hline Ambos padres & 30 & $75.0 \%$ & Ambos padres & 30 & $75.0 \%$ \\
\hline Mamá & 5 & $12.5 \%$ & Mamá & 8 & $20.0 \%$ \\
\hline Papá & 3 & $7.5 \%$ & Abuelos & 2 & $5.0 \%$ \\
\hline Tíos & 2 & $5.0 \%$ & & & \\
\hline
\end{tabular}

Tabla 2.

Categorías para demostración de afecto materno para hombres y mujeres

\begin{tabular}{|c|c|c|c|c|}
\hline \multicolumn{2}{|l|}{ Hombres } & \multicolumn{2}{|c|}{ Mujeres } & \multirow[b]{2}{*}{ Porcentaje } \\
\hline Categoría & Ejemplos & Porcentaje & Ejemplos & \\
\hline Expresión física de afecto & $\begin{array}{l}\text { "me abraza", "me besa", "es } \\
\text { cariñosa" }\end{array}$ & $40.0 \%$ & $\begin{array}{l}\text { "darme besitos", "abrazándome”, "es } \\
\text { cariñosa" }\end{array}$ & $55.0 \%$ \\
\hline Apoyo & $\begin{array}{l}\text { "me apoya en lo que no puedo", } \\
\text { "me ayuda cuando tengo } \\
\text { problemas" }\end{array}$ & $7.5 \%$ & "apoyarme en todo", "me apoya” & $12.5 \%$ \\
\hline Comprensión & $\begin{array}{l}\text { "me confía su amistad", "me } \\
\text { comprende" }\end{array}$ & $5.0 \%$ & "comprenderme", "me comprende" & $7.5 \%$ \\
\hline Expresión verbal de afecto & $\begin{array}{l}\text { "me dice te quiero", "me dice que } \\
\text { soy su orgullo" }\end{array}$ & $22.5 \%$ & $\begin{array}{l}\text { "diciéndome cuánto me quiere", "nos } \\
\text { dice que nos quiere mucho, que } \\
\text { ojalá y nunca nos pase nada" }\end{array}$ & $45 \%$ \\
\hline Cuidados y protección & $\begin{array}{l}\text { "la mayoría de las cosas que } \\
\text { hace es para que yo esté bien," } \\
\text { "me cuida" }\end{array}$ & $10.0 \%$ & $\begin{array}{l}\text { "nos da alimento, vestido y siempre } \\
\text { muestra cuidado", "tratando de } \\
\text { protegerme" }\end{array}$ & $22.5 \%$ \\
\hline Enseñanza de valores & - & - & $\begin{array}{l}\text { "nos enseña buenos valores", "me } \\
\text { corrige en lo malo que hago" }\end{array}$ & $5.0 \%$ \\
\hline Consejos & "me aconseja" & $2.5 \%$ & $\begin{array}{l}\text { "me da consejos", "me demuestra } \\
\text { que me quiere al darme consejos" }\end{array}$ & $7.5 \%$ \\
\hline Juego & "juega conmigo" & $2.5 \%$ & "juega conmigo" & $2.5 \%$ \\
\hline Expresión material de afecto & $\begin{array}{l}\text { "me compra mis libros y } \\
\text { videojuegos", "a veces me compra } \\
\text { cosas" }\end{array}$ & $10 \%$ & $\begin{array}{l}\text { "me compra cosas", "trabaja para } \\
\text { que no me falte nada" }\end{array}$ & $12.5 \%$ \\
\hline Comunicación/Tiempo & $\begin{array}{l}\text { "estoy más tiempo con ella", "habla } \\
\text { mucho conmigo", "me invita a } \\
\text { pasear" }\end{array}$ & $17.5 \%$ & $\begin{array}{l}\text { "me escucha cuando la necesito", } \\
\text { "sabe escuchar" }\end{array}$ & $7.5 \%$ \\
\hline No demuestra afecto & $\begin{array}{l}\text { "nada", "no cuando me da afecto, } \\
\text { me trata igual todo el tiempo" }\end{array}$ & $7.5 \%$ & $\begin{array}{l}\text { "no es muy afectiva", "nunca me lo } \\
\text { demuestra" }\end{array}$ & $7.5 \%$ \\
\hline
\end{tabular}


porcentaje más alto $(20.0 \%)$ son comunicación/ tiempo y expresión material de afecto, en tercer lugar se encuentra la expresión física de afecto. Las categorías con porcentajes más bajos son respeto, enseñanza de valores y juego. En el caso de las chicas, la categoría con porcentaje más alto es expresión física de afecto, seguida de la expresión verbal de afecto y la siguiente categoría con porcentaje más alto (12.5\%) es no demuestra afecto. El porcentaje más bajo corresponde a la categoría de respeto y no se reportaron respuestas para la enseñanza de valores. Cabe mencionar que una categoría que se presenta únicamente en los varones en el caso de la demostración de afecto por parte del padre, es la de regaños.

Tabla 3.

Categorías para demostración de afecto paterno para hombres y mujeres

\begin{tabular}{|c|c|c|c|c|}
\hline \multicolumn{2}{|c|}{ Hombres } & \multicolumn{2}{|c|}{ Mujeres } & \multirow[b]{2}{*}{ Porcentaje } \\
\hline Categoría & Ejemplos & Porcentaje & Ejemplos & \\
\hline Expresión física de afecto & "me abraza", "me hace cariños" & $12.5 \%$ & $\begin{array}{l}\text { "cuando voy a dormir me da un } \\
\text { beso", me abraza y me besa" }\end{array}$ & $30.0 \%$ \\
\hline Apoyo & $\begin{array}{l}\text { "me ayuda con lo que no } \\
\text { puedo, cuando no veo nada } \\
\text { cerca me hace que lo vea } \\
\text { cerca" }\end{array}$ & $7.5 \%$ & "me apoya" & $2.5 \%$ \\
\hline Comprensión & - & - & $\begin{array}{l}\text { "me comprende en los } \\
\text { problemas que le cuento", } \\
\text { "comprenderme" }\end{array}$ & $10.0 \%$ \\
\hline Expresión verbal de afecto & $\begin{array}{l}\text { "me dice que me quiere", } \\
\text { "me dice que me quiere y lo } \\
\text { orgulloso que está de mí" }\end{array}$ & $7.5 \%$ & $\begin{array}{l}\text { "me lo dice," "me dice que me } \\
\text { quiere," "me dice que está } \\
\text { orgulloso de mí" }\end{array}$ & $22.5 \%$ \\
\hline Cuidados y protección & $\begin{array}{l}\text { "me cuida", "haciendo cosas } \\
\text { buenas para mí", "se preocupa } \\
\text { por mí" }\end{array}$ & $7.5 \%$ & $\begin{array}{l}\text { "me cuida y me protege", } \\
\text { "cuidándome" }\end{array}$ & $7.5 \%$ \\
\hline Respeto & "tratarme con respeto" & $2.5 \%$ & "dándome respeto" & $2.5 \%$ \\
\hline Enseñanza de valores & $\begin{array}{l}\text { "nos hace reflexionar las } \\
\text { cosas" }\end{array}$ & $2.5 \%$ & - & - \\
\hline Consejos & $\begin{array}{l}\text { "me aconseja", "'"me da } \\
\text { consejos" }\end{array}$ & $5.0 \%$ & $\begin{array}{l}\text { "da consejos de cosas", "no } \\
\text { convivo mucho con él, así que } \\
\text { sólo me da consejos" }\end{array}$ & $10.0 \%$ \\
\hline Juego & "juega conmigo" & $2.5 \%$ & $\begin{array}{l}\text { "juega con nosotras", "juega } \\
\text { conmigo" }\end{array}$ & $5.0 \%$ \\
\hline $\begin{array}{l}\text { Expresión material de } \\
\text { afecto }\end{array}$ & $\begin{array}{l}\text { "me da dinero", "a veces } \\
\text { me compra cosas", "con } \\
\text { obsequios" }\end{array}$ & $20.0 \%$ & $\begin{array}{l}\text { "me da dinero", "me da } \\
\text { obsequios cada que me ve", } \\
\text { "me da para mis estudios" }\end{array}$ & $10.0 \%$ \\
\hline Regaños & "me regaña" & $2.5 \%$ & - & - \\
\hline Comunicación/Tiempo & $\begin{array}{l}\text { "me lleva a lugares que no } \\
\text { conozco", "platica conmigo", } \\
\text { "pasa rato conmigo" }\end{array}$ & $20.0 \%$ & $\begin{array}{l}\text { "habla conmigo, me deja } \\
\text { estar con él," "me pregunta } \\
\text { por relaciones amorosas, me } \\
\text { pregunta de la escuela" }\end{array}$ & $10.0 \%$ \\
\hline No demuestra afecto & "nada" & $5.0 \%$ & $\begin{array}{l}\text { "de vez en cuando viene, pero } \\
\text { no nos demuestra que nos } \\
\text { quiere", "nada", nada, no da } \\
\text { dinero" }\end{array}$ & $12.5 \%$ \\
\hline
\end{tabular}




\section{Discusión}

La familia puede concebirse como un sistema relacional, es decir, como un grupo de personas en interacción constante con su medio social y consigo mismas; un conjunto de necesidades determinan la manera en que se relacionan los miembros entre sí, y opera a través de pautas transaccionales. La dinámica familiar es el conjunto de dichas pautas, las cuales establecen de qué manera, cuándo y con quién se relaciona cada miembro de la familia en un momento dado, y de acuerdo al ciclo vital por el que estén atravesando. La totalidad de fuerzas, tanto positivas como negativas, determina el buen o mal funcionamiento del sistema, al cual se le atribuye una función primordial en el constante cambio y crecimiento del grupo familiar. Los efectos de la crianza o cuidado infantil en el desarrollo humano han sido reconocidos desde hace varias décadas; desde los años cuarenta, se han estudiado las actitudes y comportamientos de padres y madres, así como las interacciones padres-hijos (García, 2008).

La evidencia empírica ha revelado la validez y utilidad de la tipología de Baumrind. Se han acumulado datos donde se indica que los padres de estilo democrático, caracterizado por el afecto, el control y las exigencias de madurez, tienen hijos que muestran un mejor ajuste emocional y comportamental. Los adolescentes con padres democráticos presentan niveles más altos de autoestima y desarrollo moral, un mayor interés hacia la escuela, mejor rendimiento académico, mayor satisfacción vital, son menos conformistas ante la presión negativa del grupo de iguales, presentan menos problemas de conducta y, prácticamente, a lo largo de toda la adolescencia, consumen drogas con menor frecuencia (Rivera, Villatoro, Fleiz, Medina-Mora \& Jiménez, 1995; Parra \& Oliva, 2006; Oliva et al., 2008).

Por su parte, Gray y Steinberg (1999), mencionan que la calidez afectiva es un factor protector de carácter más general que evita este tipo de dificultades. Para Fletcher, Steinberg y
Williams-Wheeler (2004) un clima familiar caracterizado por el afecto y la cercanía emocional facilita la comunicación fluida, para que madres y padres estén informados de las actividades de sus hijos, cuestión que influiría en el menor consumo de drogas y la conducta antisocial. Además, el vínculo afectivo que el adolescente establece con sus padres lo hace más sensible y receptivo a la influencia familiar (Collins \& Laursen, 2004). Finalmente, hay que tener en cuenta lo que Noller y Callan (1988) y Smetana (1989) comentan, respecto a que padres y adolescentes tienen visiones distintas de la realidad familiar; los primeros perciben las interacciones de forma más positiva y optimista, tienden a infravalorar la tasa de conflictos y señalan más calidez y afecto que lo que indican sus hijos e hijas. A este respecto, Parra y Oliva (2006) sugieren que la opinión de los adolescentes parece estar menos influenciada por la deseabilidad social, por lo que resulta más realista y objetiva. Mientras que quienes han crecido en un ambiente indiferente tienden a presentar problemas emocionales y conductuales.

La falta de afecto y supervisión tiene efectos negativos para el desarrollo de los hijos, que con frecuencia presentan desajustes a nivel social como impulsividad, conducta delictiva o consumo abusivo de sustancias (Steinberg, 2001). También se presenta en estos jóvenes la baja autoestima y los problemas emocionales, al grado de manifestar problemas depresivos (Milevsky, Schlechter, Netter \& Keehn, 2007). Lai y McBride-Chang (2001) mencionan que mantener una relación no satisfactoria con los padres es un factor de riesgo para cometer una tentativa de suicidio. Cuando la relación entre progenitores e hijos es conflictiva se presenta un incremento del riesgo suicida. Un clima familiar positivo disminuye la probabilidad del intento de suicidio, debido a que el efecto protector sobre el comportamiento suicida en adolescentes se establece por una interacción adecuada y estable entre sus miembros. 


\section{Referencias}

Los resultados encontrados concuerdan con lo que históricamente conocemos: las manifestaciones afectivas por lo general se asocian al papel de la madre, mientras que el padre, con cierta distancia afectiva (Laqueur, 1992); sin embargo, se encontró una buena cantidad de chicos y chicas que mencionó que su padre demuestra su afecto con caricias.
Baumrind, D. (1968). Authoritarian vs. authoritative parental control. Adolescence, 3, 255-272.

Collins,W. A. \& Laursen, B. (2004). Parent-adolescent relationships and influences. En R.M. Lerner \& L. Steinberg (Eds.), Handbook of adolescent psychology (pp. 331-361). Nueva Jersey: Willey.

Dishion, T., Patterson, G., Stoolmiller, M. \& Skinner, M. (1991). Family, school, and behavior antecedents to early adolescent involvement with antisocial peers. Developmental Psychology, 27, 172-180.

Dusek, B. \& Mcintyre, G. (2003). Self-concept and self-esteem development. En G. R. Adams \& M. D. Berzonsky (Eds.), Blackwell Handbook of Adolescence (pp. 290-309). Malden: Blackwell Publishing.

Fletcher, C., Steinberg, L. \& Williams-Wheeler, M. (2004). Parental Influences on Adolescent Problem Behavior: Revisiting Stattin and Kerr. Child Development, 75, 781-796.

Flouri, E. \& Buchanan, A. (2002). What predicts good relationships with parents in adolescence and partners in adult life: Findings from the 1958 British birth cohort. Journal of Family Psychology, 16, 186-198.

Frauenglass, S., Routh, D., Pantin, H. \& Mason, C. (1997). Family support decreases influence of deviant peers on Hispanic adolescents substance use. Journal of Clinical Child Psychology, 26, 15-23.

García, L. (2008). Niñez: Pautas de crianza y escolaridad en familias migrantes voluntarias de sectores populares de Cali, Colombia. Pensando Psicología, 4(6), 18-27.

Gray, R. \& Steinberg, L. (1999). Unpacking Authoritative parenting: Reassessing a Multidimensional Construct. Journal of Marriage and The Family, 61, 574-588.

Hernández, S., Fernández, C. \& Baptista, L. (2008). Metodología de la investigación. México: Mc Graw- Hill

Khaleque, A. \& Rohner, P. (2002). Perceived parental acceptancerejection and psychological adjustment: A metanalisis of crosscultural and intracultural studies. Journal of Marriage and the Family, 64, 54-64.

Lai, W. \& McBride-Chang, C. (2001). Suicidal ideation, parenting style and family dimate among Hong Kong adolescents. International Journal of Psychology, 36, 81-87.

Laqueur, T. (1992). Los hechos de la paternidad. Debate Feminista, 6, 14-17.

Maccoby, E. \& Martín, J. (1983). Socialization in The Context of the family Parent-child interactions. En E. M. Hetherington y P. H. Mussen (Eds.), Handbook of child Psychology (vol. 4, pp. 1-101). Nueva York: Wiley.

Martínez, J.L., Fuertes, A., Ramos, A. \& Hernández, A. (2003). Consumo de drogas en la adolescencia: importancia del afecto y la supervisón parental. Psicothema, 15(2), 161-166.

Martínez, J.M. \& Robles, L. (2001). Variables de protección ante el consumo de alcohol y tabaco en la adolescencia. Psicothema, 13(2), 222-228.

Mestre, M. V., Frías, D., Samper, P. \& Nácher, M. J. (2003). Estilos de crianza y variables personales como factores de riesgo de la conducta agresiva. Revista Mexicana de Psicología, 20(2), 189-199.

Milevsky, A., Schlechter, M, Netter, S. \& Keehn, D. (2007). Maternal and paternal parenting styles in adolescents: Associations with selfesteem, depression and life-satisfaction. Journal of Child and Family Studies, 16, 39-47.

Motrico, E., Fuentes, M. J. \& Bersabé R. (2001). Discrepancias en la percepción de los conflictos entre padres e hijos/as a lo largo de la adolescencia. Anales de Psicología, 17 (1), 1-13.

Noller, P. \& Callan, V. J. (1988). Understanding parentadolescent interaction: the perception of family members and outsiders. Developmental Psychology, 24, 707-714.

Oliva, A. (2006). Relaciones familiares y desarrollo adolescente. Anuario de Psicología, 37, 209-224.

Oliva, A., Parra, A. \& Sánchez-Queija. (2002). Relaciones con padres e iguales como predictoras del ajuste emocional y conductual durante la adolescencia. Apuntes de Psicología, 20, 3-16. 
Oliva, A., Parra, A. \& Arranz, E. (2008). Estilos relacionales parentales y ajuste adolescente. Infancia y aprendizaje, 31(1), 93-106.

Parra, A. \& Oliva, A. (2002). Comunicación y conflicto familiar durante la adolescencia. Anales de Psicología, 18(2), 215-231.

Parra, A., Oliva, A. \& Sánchez-Queija, I. (2004). Evolución y determinantes de la autoestima durante los años adolescentes. Anuario de Psicología, 35, 331-346.

Parra. A. \& Oliva, A. (2006). Un análisis longitudinal sobre las dimensiones relevantes del estilo parental durante la adolescencia. Infancia y Aprendizaje, 29, 453-470.

Rivera, E., Villatoro, J., Fleiz, C., Medina-Mora, M. \& Jiménez, A. (1995). Percepción de las características de los padres y su relación con el consumo de drogas. Revista de Psicología Social y Personalidad, $11,149-158$.

Ramírez, M.A. (2005). Padres y desarrollo de los hijos: Prácticas de crianza. Estudios Pedagógicos, 31(2), 167-177. doi: 10.4067/S071807052005000200011

Rodrigo, M.J., Máiquez, M.L., García, M., Mendoza, R., Rubio, A., Martínez, A. \& Martín, J.C. (2004). Relaciones padres-hijos y estilos de vida en adolescentes. Psicothema, 16(2), 203-210.

Serrano, G., Godás, A., Rodríguez, D. \& Mirón, L. (1996). Perfil psicosocial de los adolescentes españoles. Psicothema, 8(1), 25-44.

Smetana, J. G. (1989). Adolescents' and parents' reasonig about family conflict. Child Development, 60, 1052 -1067.

Steinberg, L. (2001). We know some things: parent-adolescent relationships in retrospect and prospect. Journal of Research on Adolescence, 11, 1-19.

Von der Lippe, A. (1998). Are conflict and challenge sources of personality development. En E. Sokoe \& A. von der Lippe (Eds.), Personality development in adolescence: $A$ cross national and life span perspective (pp. 38-60). Londres: Routledge. 